\title{
Regeneración natural y patrones de distribución espacial de la palma chilena Jubaea chilensis (Molina) Baillon en los bosques mediterráneos de Chile central
}

\section{Natural regeneration and spatial distribution patterns of Chilean palm Jubaea chilensis (Molina) Baillon in Mediterranean forests of Central Chile}

\author{
Alejandro Miranda ${ }^{1,2^{*}}$, H. Jaime Hernández ${ }^{3}$, Ramiro Bustamante ${ }^{4}$, E. Matías Díaz ${ }^{5}$, L. Alberto \\ GonzÁlez ${ }^{6}$ \& Adison Altamirano ${ }^{1}$
}

${ }^{1}$ Universidad de La Frontera, Departamento de Ciencias Forestales, Laboratorio de Ecología del Paisaje Forestal, Casilla 54-D, Temuco, Chile.

${ }^{2}$ Escuela de Graduados, Facultad de Ciencias Forestales y Recursos Naturales, Universidad Austral de Chile, Casilla 567, Valdivia, Chile.

${ }^{3}$ Laboratorio de Geomática y Ecología del Paisaje, Facultad de Ciencias Forestales y de la Conservación de la Naturaleza, Universidad de Chile, Av. Santa Rosa 11.315, Santiago, Chile.

${ }^{4}$ Departamento de Ciencias Ecológicas, Instituto de Ecologia y Biodiversidad, Facultad de Ciencias, Universidad de Chile, Las Palmeras 3425, Ñuñoa, Santiago, Chile.

${ }^{5}$ Corporación Nacional Forestal, Región Metropolitana de Santiago. Sección Fiscalización Forestal y Evaluación Ambiental, Paseo Bulnes 285, Santiago, Chile.

${ }^{6}$ Universidad de Chile, Departamento de Gestión Forestal y su Medio Ambiente, Santa Rosa 11.315, La Pintana, Santiago.

*alejandro.miranda@ufrontera.cl

\begin{abstract}
RESUMEN
Jubaea chilensis es un árbol endémico de la zona central de Chile $\left(31^{\circ} 15^{\prime}-35^{\circ} 22^{\prime} \mathrm{S}\right)$. Las principales amenazas actuales a su conservación estarían asociadas a diferentes limitantes a su regeneración natural, en especial la intensa cosecha de sus semillas. El análisis de patrones espaciales de distribución puede ser una importante herramienta para entender la dinámica de los bosques y la interacción entre los individuos. En este trabajo se estudió el patrón espacial de reclutamiento de la especie y se identificaron las condiciones favorables para su regeneración. Se estimó una notoria agrupación espacial entre los individuos de regeneración para todos los rangos de distancia analizados, y a su vez una distribución dependiente de los individuos adultos, agrupándose mayormente cercano a ellos. Por otra parte, la modelación espacial reveló que la regeneración natural de $J$. chilensis se relaciona significativamente con sitios de mayor cobertura vegetacional, y especialmente en sitios con una mayor radiación solar. Los resultados sugieren que la regeneración agrupada de J. chilensis se debería a la asociación de condiciones ambientales propicias para el establecimiento de nuevos individuos, su mecanismo de dispersión de semillas y escape de la depredación, herbivoría y cosecha de sus semillas, lo que estaría determinando la estructura espacial de la población. A su vez, los resultados confirman un efecto nodriza, facilitador de la regeneración natural de la especie.
\end{abstract}

Palabras Clave: Modelos espacialmente explícitos, efecto nodriza, clima mediterráneo, herbivoría.

\begin{abstract}
Jubaea chilensis is an endemic tree of Central Chile $\left(31^{\circ} 15^{\prime}-35^{\circ} 22^{\prime} \mathrm{S}\right)$. The current main conservation threats for this species should be associated with different factors which limit its natural regeneration, in particular the intense seeds crop. The analysis of spatial distribution patterns can be an important tool to understand the forest dynamic and the individual interactions. In this study was studied the spatial pattern of species recruitment and identifying the proper conditions for its regeneration. We predicted a clear spatial clumped pattern between regeneration individuals for all distance range studied, and also a clumped distribution associated to adult individuals. Spatial modeling showed that natural regeneration of $J$. chilensis is significantly related to higher vegetation cover, and especially in those with higher solar radiation. The results suggest that clumped regeneration of $J$. chilensis could be explained by the associated environmental conditions promoting the establishment of new seedlings, its seed dispersal mechanism, predation avoid, herbivory, and seeds crop. We also found a nurse effect which facilitates the natural regeneration of this species.
\end{abstract}

KEYWORDS: Spatially explicit models, nurse effect, Mediterranean climate, hervibory. 


\section{INTRODUCCIÓN}

La palma chilena (Jubaea chilensis (Molina) Baillon) es un árbol monotípico, amenazado y endémico de Chile (González et al. 2009). Se distribuye entre la región de Coquimbo $\left(31^{\circ} 15^{\prime} \mathrm{S}\right)$ y la localidad de Tapihue ( $\left.35^{\circ} 22^{\prime} \mathrm{S}\right)$, en Chile central. Esta distribución es discontinua y altamente fragmentada por acción antrópica (González et al. 2009). Se estima que su población actual es de 120.000 individuos en distintos estados de desarrollo, lo que representaría aproximadamente un 2,5\% de su población original (González et al. 2009). Esta especie ha visto reducida sus poblaciones en parte, por su explotación para la producción de miel de palma y por la extensiva deforestación ocurrida en la zona central de Chile (Cabello 2006). Sin embargo, actualmente existe consenso que las principales amenazas para su conservación se relacionan con diferentes factores antrópicos que limitan tempranamente su regeneración natural (Cabello 2006, Marcelo et al. 2006, González et al. 2009).

Es ampliamente validado que para la recuperación exitosa de cualquier especie vegetal, es clave entender su dinámica regenerativa, y en particular conocer los factores ecológicos que facilitan o limitan su regeneración natural (Jordano et al. 2004). La regeneración de un nuevo individuo es un proceso donde se deben superar diferentes barreras que condicionan la producción de flores y frutos, la dispersión y germinación de semillas, sobrevivencia de plántulas y el desarrollo hasta llegar al estado adulto (Jordano et al. 2004). El conjunto de estos procesos ecológicos en su interacción con el medio ambiente tienen una expresión particular, determinando la distribución espacial de los individuos en diferentes estados de desarrollo (Law et al. 2002). Por ejemplo, la dispersión de la semilla determina el rango de distancia en los cuales podría reclutar un individuo desde la planta madre (Cousens et al. 2008). Sin embargo, el reclutamiento en ese rango de distancia se expresará en la medida que exista una concordancia espacial entre la sombra de semillas y un hábitat favorables para el desarrollo de un nuevo individuo (Nathan \& Muller-Landau 2000, Getzin et al. 2014). En ambientes muy adversos, esta concordancia entre la sombra de semillas y condiciones ecológicas para el reclutamiento puede no ocurrir, pudiendo incluso limitar la regeneración de las especies (Gómez-Aparicio 2008).

En ecosistemas mediterráneos, es común que los patrones espaciales de la regeneración sean reflejo de la interacción planta-planta. Ejemplo de ello es el efecto nodriza, donde un individuo genera las condiciones apropiadas para la germinación y establecimiento de otro, pudiendo ser de la misma u otra especie (Holmgren et al. 2000, GómezAparicio et al. 2004, Gómez-Aparicio 2008). Este tipo de interacción planta-planta genera un patrón agrupado de la regeneración natural en función a la distribución de los individuos que actúan como nodriza, especialmente en ambientes donde puede ser muy improbable la regeneración fuera de ellas (Fuentes et al. 1984, 1986, Holmgren et al. 2000). Por otro lado, este positivo efecto podría anularse en condiciones extremas, donde los individuos pueden competir por los recursos disponibles (Holmgren et al. 1997). Esto último podría expresarse espacialmente como un patrón de distribución regular entre individuos de la misma u otra especie, como una expresión espacial de la competencia por recursos (Palik et al. 2003).

Los mecanismos que afectan la sobrevivencia de las plantas en diferentes estados del ciclo de vida se pueden expresar en los cambios en la densidad poblacional. La densidad de individuos puede variar en función de factores bióticos (competencia intra e interespecífica), condiciones climáticas, atributos del paisaje o bien de perturbación humana (Hamill \& Wright 1986, Palik et al. 2003, Fuentes-Castillo et al. 2012). La variación espacial en la disponibilidad de agua (Ferreira et al. 2007), las propiedades físicas del suelo (Alameda et al. 2012), características topográficas (Zhang et al. 2010), cobertura vegetacional (Gómez-Aparicio 2008), estructura y composición del bosque (Donoso \& Nyland 2005), entre otros factores que pueden también afectar la densidad a la cual ocurre la regeneración.

En la zona central de Chile, las condiciones climáticas locales o la protección de las plántulas en sus primeras etapas de desarrollo, debido a las prolongadas sequías y altas temperaturas de verano, y la herbivoría, pueden generar condiciones favorables para la regeneración natural (Fuentes et al. 1984, Newton \& Tejedor 2011). Variables como la exposición topográfica, pendiente, presencia de cursos permanentes de agua o la cobertura, pueden ser determinantes en el éxito y densidad de reclutamiento de las especies (Fuentes-Castillo et al. 2012).

Uno de los principales factores que limita la regeneración natural de $J$. chilensis sería la masiva cosecha de sus semillas para el consumo humano (González et al. 2009). La reducción de cobertura vegetal, debido al intensivo cambio de uso de suelo y continuos incendios forestales en la región (Quintanilla \& Castillo 2009, Schulz et al. 2010), también afecta la regeneración natural disminuyendo los microhábitat disponibles para la supervivencia de semillas y plántulas (Marcelo et al. 2006, González et al. 2009). Además, si bien los incendios forestales, dependiendo de su severidad, no afectarían significativamente a los individuos adultos (González \& Vita 1987), la mortalidad de plántulas y la eliminación de la cubierta nodriza se vería negativamente afectada. Otras limitantes ecológicas son la herbivoría, la depredación de semillas y la mortalidad de plántulas por desecación (Yates et al. 1994, Marcelo et al. 2006). En esa vía, experimentos de campo han observado una mortalidad de $70 \%$ a causa de la herbivoría, lo que resulta especialmente limitante para la regeneración de $J$. chilensis, ya que no regenera vegetativamente (Cabello 2006). 
Una primera aproximación para entender el proceso de regeneración natural es mediante la determinación de la interacción espacial entre los individuos de regeneración, o de la regeneración en función a los adultos. La distancia a la cual se encuentra un individuo de otro puede ser reflejo del mecanismo de dispersión de semillas, depredación, facilitación, competencia, entre otros, o debido a limitaciones del ambiente, lo cual definirá la estructura espacial de la población. En segundo lugar, entender cómo la heterogeneidad espacial afecta la regeneración natural, permite delimitar condiciones en las cuales este proceso se pueda ver favorecido o limitado, evidenciando relaciones de los individuos con su medio ambiente. Considerando las limitantes para el reclutamiento y desarrollo de J. chilensis, nos preguntamos como éstas pueden afectar la estructura espacial de la población y la distribución espacial de la regeneración. Por lo tanto, nuestros objetivos son: i) explorar la interacción espacial entre individuos en diferentes estados de desarrollo (especialmente adulto-regeneración) y ii) determinar los factores ecológicos que determinan la densidad poblacional.

\section{MATERIALES Y MÉTODOS}

ÁREA DE ESTUDio

El estudio se realizó en el sector Palmas de Ocoa del Parque Nacional la Campana ( $35^{\circ} 55^{\prime} \mathrm{S}, 71^{\circ} 5^{\prime} \mathrm{W}$ ) (Fig. 1). El área se ubica dentro del tipo climático mediterráneo, caracterizado por la existencia de una estación lluviosa en invierno y por una estación seca más prolongada. Pertenece a la formación vegetacional del bosque esclerófilo mediterráneo costero de Lithrea caustica (Molina) Hook. et Arn. y Cryptocarya alba (Molina) Looser, la cual es caracterizada por la dominancia de Lithaea caustica asociado principalmente a Cryptocarya alba, Peumus boldus y Schinus latifolius (Gill. ex Lindl.) Engler (Luebert \& Pliscoff 2005). En el Parque Nacional se encuentra la población de $J$. chilensis de mayor tamaño y es la que se considera en mejor estado de conservación en toda su distribución geográfica (González et al. 2009).

El sector del palmar de Ocoa corresponde a una micro cuenca con exposición predominantemente noreste ubicada en la vertiente oriental de la Cordillera de la Costa, con

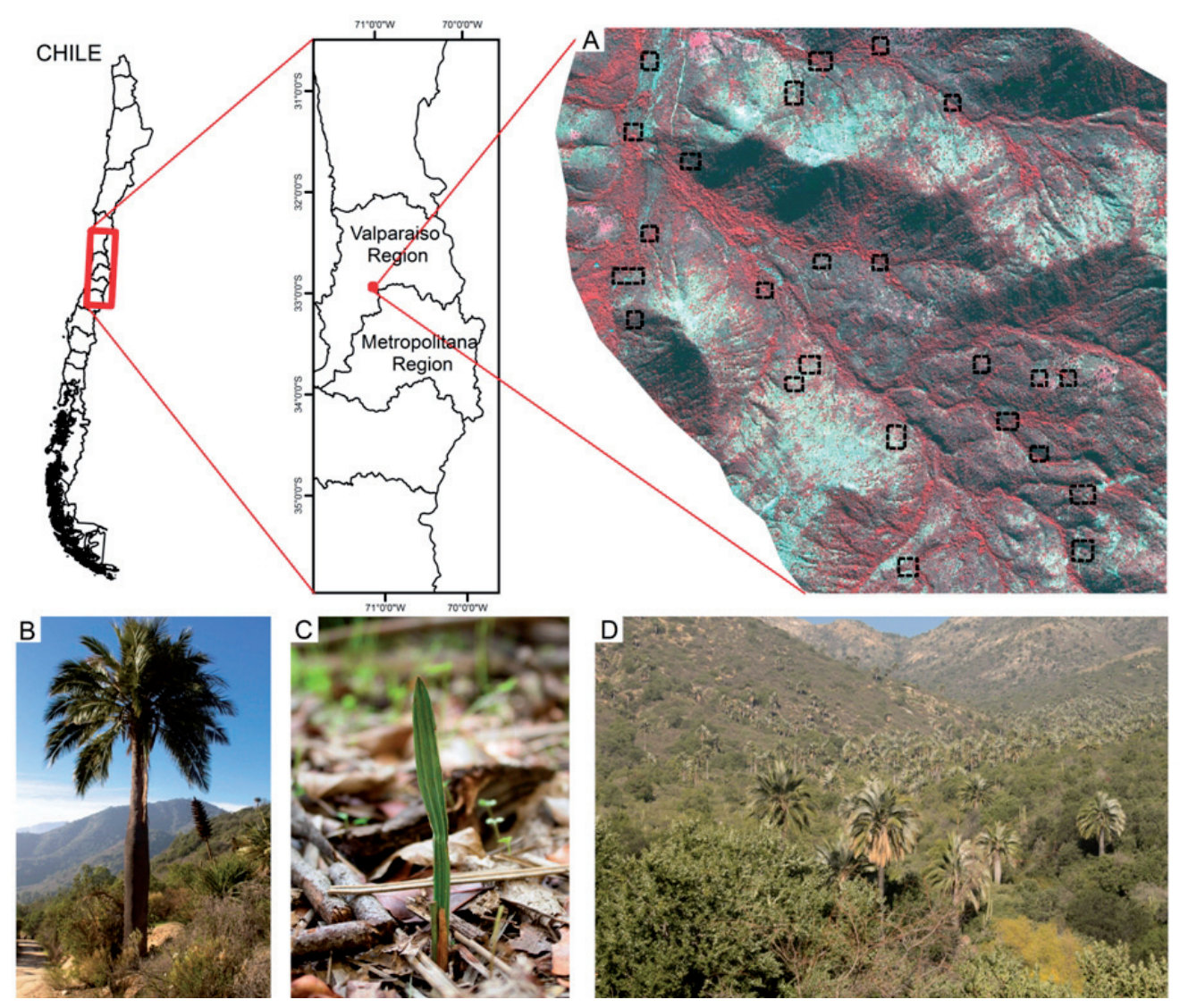

Figura 1. A. Imagen QuickBird en composición falso color estándar del palmar de Ocoa, en el Parque Nacional La Campana y distribución espacial de las parcelas de muestreo. B. Individuo adulto de Jubaea chilensis. C. Plántula de J. chilensis. D. Fotografía palmar de Ocoa.

FiguRE 1. A. QuickBird imagen in standard false color composition of Ocoa palms in La Campana National Park, and spatial distribution of sampling field plots. B. Adult of Jubaea chilensis. C. Seedling of J. chilensis. D. Photography of Ocoa palmar. 
precipitaciones aproximadas de $300 \mathrm{~mm} / \mathrm{año}$. En este sector habría aproximadamente 80.000 individuos de la especie (Hechenleitner et al. 2005), encontrándose distribuidos entre los $400 \mathrm{y}$ los $1.400 \mathrm{msnm}$. A pesar de ser un área protegida, se realiza una extracción de semillas para el consumo humano que en algunos casos puede llegar a valores cercanos al 100\% (González obs. pers.). Esto, sumado a la herbivoría y otros factores que puedan estar afectando la regeneración de la especie, hacen que la mayor parte de las subpoblaciones presenten estructuras dominadas por individuos adultos, estimándose una baja regeneración (aprox. 10 plántulas por ha para el sector de Ocoa, Michea 1988).

\section{DATOS}

Se realizó un muestreo estratificado aleatorio en un área de aproximadamente 1.300 ha. El área se dividió en microcuencas y se consideró que cada cual tenga al menos una parcela de muestreo. En total se instalaron 24 parcelas de muestreo de 100x100 m (Fig. 1) asignadas al azar dentro de cada microcuenca. Este gran esfuerzo de muestreo se justifica por la baja densidad de regeneración esperada, y la necesidad de abarcar la heterogeneidad ambiental del sector. En cada parcela se determinó la posición geográfica de cada individuo, utilizando un sistema de coordenadas local definido en terreno, con coordenadas $\mathrm{x}$ e y entre $0 \mathrm{y}$ $100 \mathrm{~m}$. Luego, mediante el posicionamiento geográfico del punto $(0,0)$ por medio de un GPS, se transformó cada coordenada relativa a un sistema de coordenadas estándar, específicamente transformado a WGS84 UTM Zona 19s. La posición geográfica de los individuos adultos fue corroborada en una imagen QuickBird II, con lo que se mantiene la posición relativa con los individuos en menor estado de desarrollo. En terreno, cada individuo de $J$. chilensis encontrado al interior de la parcela se caracterizó en función de su estado de desarrollo en: i) individuos adultos: definidos así por encontrarse en el periodo reproductivo, $\mathrm{Y}$ ii) individuos de regeneración: correspondientes a los individuos que se encuentran en estados de desarrollo tempranos, incluyendo individuos de regeneración e infantiles según la categorización de Michea (1988).

DisTRIBUCIÓN ESPACIAL DE INDIVIDUOS EN DIFERENTES ESTADOS DE DESARROLLO

Para caracterizar el patrón de distribución espacial de la regeneración de $J$. chilensis, se utilizó la función $\mathrm{K}$ de Ripley $(K(r))$ (Ripley 1981), que corresponde a uno de los métodos más utilizados en el análisis de la interacción espacial entre plantas (Pelissier \& Goreaud 2001, Wiegand \& Moloney 2004, Rozas \& Camarero 2005, Law et al. 2009). Este método permite detectar la estructura espacial de una población (agregada, aleatorio o regular), intensidad (qué tan agregado o regulares están los individuos) y rango espacial (entre qué rangos de distancias se producen estas estructuras).
Se realizó un análisis univariante entre los individuos de regeneración, y un análisis bivariante para describir la interacción espacial de los individuos de regeneración en función de los adultos. Para el cálculo de $K(r)$, se utilizó una distancia máxima de $25 \mathrm{~m}$, debido a posibles errores de interpretación en distancias mayores a 1/3 del lado de la parcela de menor longitud (Baddeley \& Turner 2005). Para la interpretación de los resultados se utilizó la transformación $L$ (r) propuesta por Besag (1977). De esta manera, se generaron intervalos de confianza que permiten comparar visualmente el patrón observado versus un patrón aleatorio para el análisis univariado o independiente en el caso del bivariado. Si el patrón observado supera el intervalo de confianza superior, se interpreta como un agrupamiento de los individuos. Y si el patrón observado está bajo el límite inferior, se interpreta como una distribución agregada.

VARIACIÓN DE LA DENSIDAD POBLACIONAL EN FUNCIÓN A LA HETEROGENEIDAD AMBIENTAL

Se construyó un modelo estadístico en donde se relacionó la densidad de individuos de regeneración (variable respuesta) con diferentes variables ecológicas (variables predictoras). Se construyeron archivos digitales en formato raster para todas las variables ecológicas con una resolución espacial de $5 \mathrm{~m}$. Se construyeron variables comúnmente utilizadas en análisis espacial y que pueden potencialmente influir en la dinámica de regeneración natural de $J$. chilensis. Las variables utilizadas fueron: i) elevación, ii) distancia a los fondos de quebrada, iii) exposición topográfica, iv) pendiente v) índice de humedad topográfico, vi) distancia a individuo adulto más cercano vii) densidad de individuos adultos, y viii) índice de diferencia normalizado de vegetación (NDVI). Cada variable o una combinación de ellas podrían revelar mecanismos ecológicos mediante los cuales se pueda favorecer o inhibir la regeneración natural de la especie. En particular, se consideró que estas variables son proxis de la accesibilidad a cada rodal, la disponibilidad de recursos hídricos, la radiación solar y los valores NDVI como una aproximación al efecto nodriza que ejerce la cobertura vegetal.

Para la construcción de las variables descritas se generó un modelo digital de elevación mediante la restitución de pares estereoscópicos de fotografías aéreas (Lillesand et al. 2008). Para ello se utilizó el módulo Imagine OrthoBase implementado en el software Erdas 8.6 (ERDAS 2002). Las fotografías utilizadas fueron adquiridas por el vuelo FONDEF del año 1994 a una escala 1:20.000.

La pendiente y la exposición fueron calculadas utilizando el módulo Spatial Analyst implementado en el software Arcgis 9.3. El índice de humedad topográfico (TWI) es un indicador del potencial de disponibilidad de agua (Beven \& Kirkby 1979, O’Loughlin 1981). Este índice se calculó como el ln $(\mathrm{a} / \tan \mathrm{b})$, donde "b" es la pendiente de la superficie analizada y "a" es la superficie aportante 
sobre cada punto, calculado como a $=\mathrm{A} / \mathrm{L}$, donde $\mathrm{A}\left(\mathrm{en}^{2}\right.$ ) es la superficie aportante sobre cada punto y L (en metros) es la longitud de la isolínea. Los valores mayores de TWI representan zonas relativamente planas con grandes áreas aportantes de humedad (fondos de quebrada), donde es esperable encontrar una mayor disponibilidad de agua que en zonas con pendientes mayores o con menores superficies aportantes (Wilson \& Gallant 2000). Para el cálculo de la distancia desde los fondos de quebradas, primero fue necesario calcular las zonas de acumulación hídrica, delineando los fondos de quebradas con una superficie mínima de área de acumulación de una hectárea. Este análisis se realizó en el módulo Hydrology implementado en el software Arcgis 9.3. La distancia a los fondos de quebrada fue calculada utilizando el módulo Spatial Analyst implementado en el software Arcgis 9.3.

Para representar el grado de cobertura vegetal se utilizó el NDVI. Para esto, una imagen QuickBird II fue preprocesada a través de la fusión de imágenes en el software ERDAS 8.6. Con esto, se obtuvo una imagen multiespectral con una resolución espacial de 0,6 metros. Posteriormente, la imagen QuickBird II fue transformada a radiancia mediante procedimientos estándar Krause (2003). Se calculó el NDVI utilizando la formula (NIR-R)/(NIR+R), donde NIR corresponde a la banda espectral ubicada en el rango espectral del infrarrojo cercano $(760-900 \mathrm{~nm})$, y R es la banda ubicada en el rojo (630-690 nm). Los valores más altos representan vegetación sana (NDVI $>0,3$ ), con una mayor actividad fotosintética y los valores menores representarían zonas con menos cantidad de vegetación (Lillesand et al. 2008),

Para la construcción del modelo estadístico se utilizaron los datos de las 24 parcelas prospectadas en terreno. El modelo general construido es equivalente a un modelo lineal generalizado, donde la variable respuesta es la densidad de individuos por unidad de área y las variables predictoras, todas las variables espacialmente explícitas mencionadas anteriormente (Baddeley \& Turner 2005). Para el ajuste se utilizó una distribución de Poisson, que corresponde a la hipótesis de la completa aleatoriedad espacial. Debido a la posible relación de la densidad de individuos con variables ambientales, se utilizó un modelo que puede dar cuenta de un proceso no homogéneo de Poisson, donde la densidad es dependiente de la variabilidad ambiental (Baddeley \& Turner 2005). Se compararon 18 modelos con diferentes combinaciones de variables ecológicas.

El proceso no homogéneo de Poisson es utilizado para la modelación de procesos puntuales en ambientes heterogéneos (Penttinen \& Stoyan 2000). La heterogeneidad espacial es expresada mediante las diferentes variables espacialmente explícitas, las cuales pueden ser reflejo de distintos procesos ecológicos o perturbaciones que han ocurrido y que han permitido que se establezca un individuo. La inclusión de estas variables puede dar señales de las condiciones ecológicas en las cuales se favorece el proceso de establecimiento de individuos de la especie. Las variables son expresadas como imágenes que son utilizadas en el ajuste del modelo, incluidas como una lista asociada al patrón de puntos. El procedimiento se realizó en el paquete SPATSTAT implementado en el software R (Baddeley \& Turner 2005).

La selección del modelo final se realizó utilizando el índice de información de Akaike (AIC) y el índice de correlación entre los valores observados y predichos por cada uno de los modelos, en donde se contrastó la densidad de individuos por unidad de área. De esta manera se seleccionó el modelo que mayormente se ajusta a las observaciones realizadas en terreno. Las variables predictoras seleccionadas por el modelo con el menor AIC y mayor correlación serán las consideradas como relevantes en la configuración del nicho de regeneración de la especie.

\section{RESULTADOS}

En las veinticuatro parcelas prospectadas en terreno se encontraron 300 individuos adultos y 229 de regeneración. La densidad media de regeneración fue de 9,6 ind/ha, con un rango de 0 a 22,7 ind/ha (Fig. 2). En tres parcelas no se encontró regeneración.

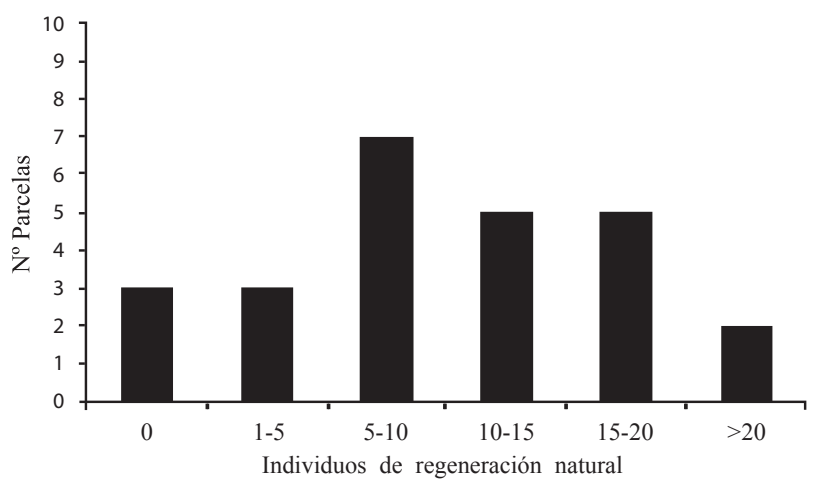

Figura 2. Distribución del número de individuos de regeneración por cada parcela de muestreo.

FIGURE 2. Distribution of the number of regeneration individuals for each sampling field plot.

DisTRIBUCIÓN ESPACIAL DE INDIVIDUOS EN DIFERENTES ESTADOS DE DESARROLLO

Todos los patrones espaciales analizados -interacción entre individuos adultos, interacción entre individuos de regeneración y de los individuos de regeneración en función a los adultos- fueron significativamente diferentes a un patrón de distribución aleatorio $(\mathrm{p}<0,001)$. Se observó una notoria agrupación espacial entre los individuos de regeneración (juveniles) para todos los rangos de distancia analizados (Figura 3 a), con agrupamiento relativamente 
constante desde los $5 \mathrm{~m}$. Esto implica que para todos los rangos de distancia analizados se detecta una agregación de la regeneración. Este patrón se repite para los individuos adultos, pero con una menor intensidad que lo observado para individuos juveniles (Fig. 3 b).
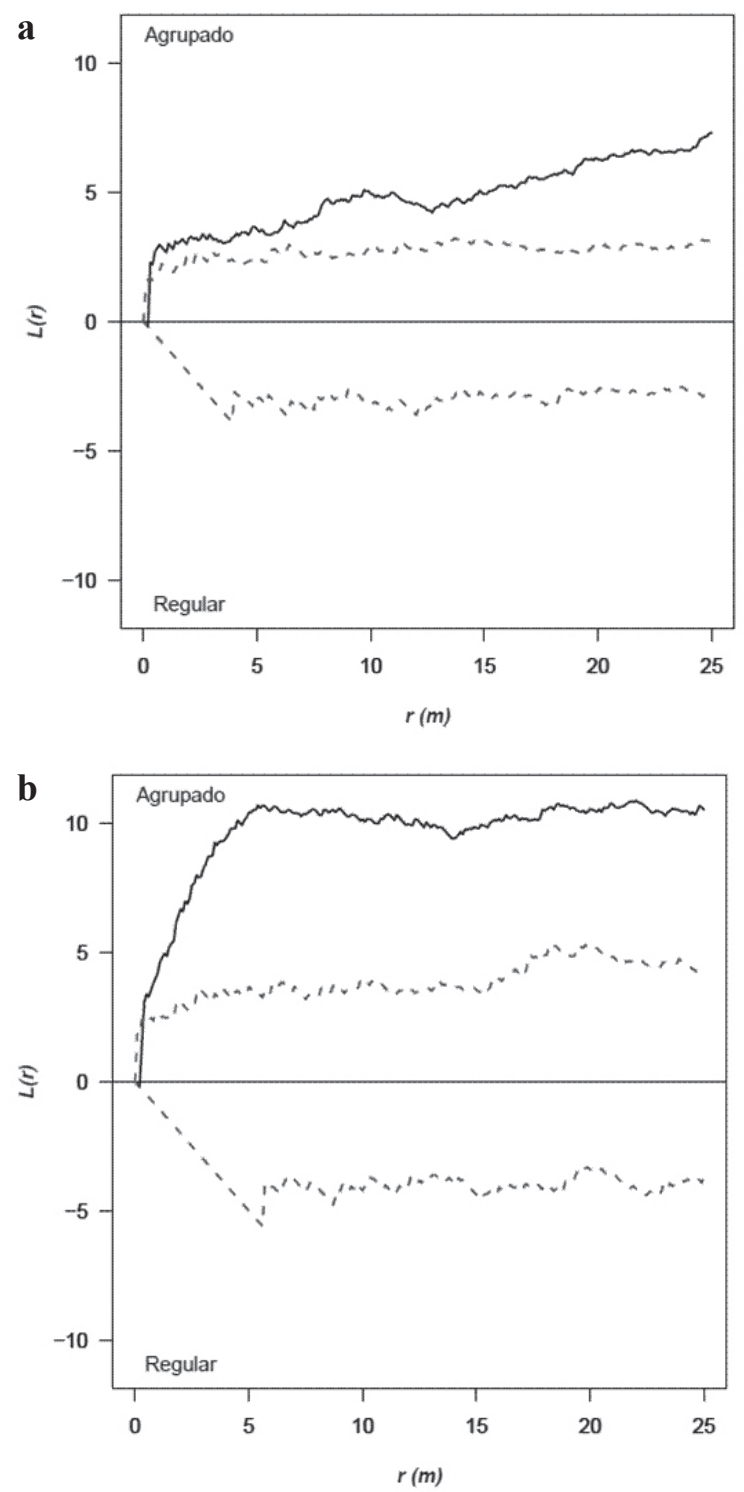

FIGURA 3. Valores de $L(r)$ entre individuos de Jubaea chilensis para diferentes escalas espaciales: a) entre individuos de regeneración y b) entre individuos adultos, siendo $r$ la distancia de interacción. La línea continua corresponde al patrón observado; la línea discontinua representa el intervalo de confianza del $95 \%$ de una distribución espacial al azar.

FIgURE 3. Values of $L(r)$ between individuals of Jubaea chilensis for different spatial scales: a) between regeneration individuals, b) between adult individuals. $r$ is the interaction distance. Continued lines represent the observed pattern; dashed lines represent is the confident interval of $95 \%$ for a random spatial distribution.
El patrón observado de la interacción espacial entre los individuos de regeneración y los adultos (análisis bivariado) muestra una distribución dependiente de la regeneración hacia los individuos adultos, habiendo una mayor densidad de regeneración en torno a los individuos adultos (Fig. 4). Sin embargo, esta interacción es más intensa en un intervalo entre los 5 y $7 \mathrm{~m}$, donde se observa la mayor densidad de individuos de regeneración. El 48\% de los individuos de regeneración se encuentran a menos de $8 \mathrm{~m}$ de un posible árbol madre. Por otra parte, el patrón observado muestra una interacción a cortas distancias que no difiere, o es muy similar, a una distribución aleatoria hasta aproximadamente los $2 \mathrm{~m}$ desde la planta madre. El agrupamiento de la regeneración hacia los adultos se mantiene a mayores rangos de distancia, en donde es relativamente constante luego de los $12 \mathrm{~m}$.

VARIACIÓN DE LA DENSIDAD POBLACIONAL EN FUNCIÓN A LA HETEROGENEIDAD AMBIENTAL

El modelo seleccionado retuvo tres de las ocho variables utilizadas inicialmente como predictoras: distancia a individuo adulto más cercano, NDVI y la exposición (nortesur), todos con parámetros altamente significativo $(p<0,001)$ (Tabla I).

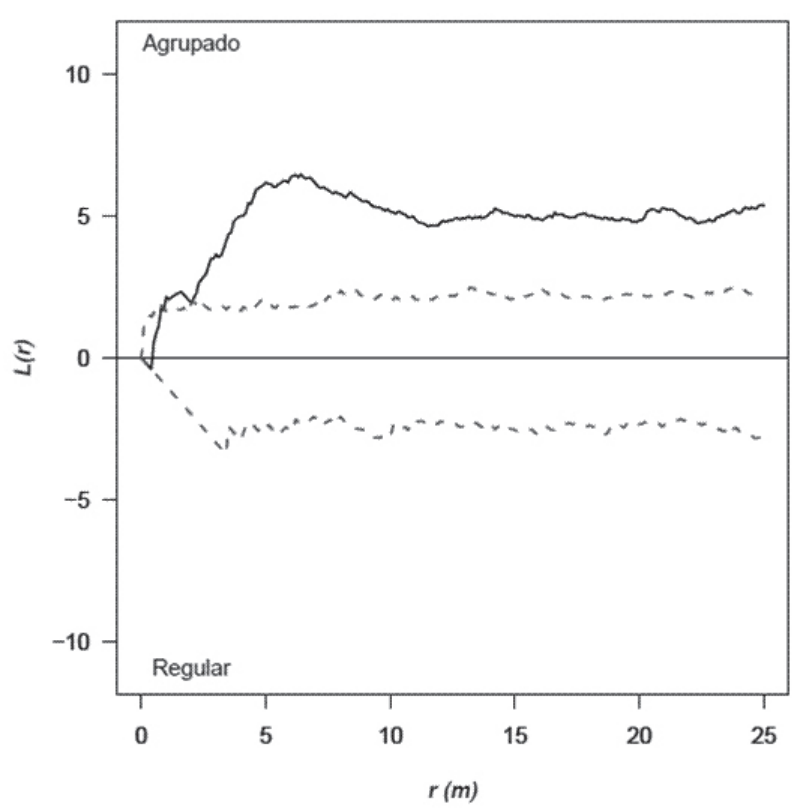

FIgURA 4. Estadístico $L(r)$ de la interacción espacial entre individuos de regeneración en función a los adultos de Jubaea chilensis, siendo $r$ la distancia de interacción. La línea continua corresponde al patrón observado; la línea discontinua representa el intervalo de confianza $>95 \%$ para un proceso espacial aleatorio.

FiguRE 4. $L(r)$ statistic of spatial interaction between regeneration individuals and adults of Jubaea chilensis., $r$ is the interaction distance. Continued lines represent the observed pattern; dashed lines represent the confident interval of $95 \%$ for a random spatial distribution. 
El modelo seleccionado predice que la mayor densidad de regeneración natural ocurriría a cortas distancias de los individuos adultos, en sitios con mayor cobertura vegetacional, y especialmente en sitios con una mayor radiación solar-relación positiva entre densidad y exposición topográfica norte-. El modelo a su vez predice correctamente la presencia de al menos un individuo de regeneración y también las zonas en las cuales la regeneración natural de la especie estaría ausente. La presencia/ausencia de individuos de regeneración es predicha acertando en un $61 \%$ de la presencia de al menos un individuo de regeneración y en un $80 \%$ de las ausencias.

TABLA I. Evaluación de modelos inhomogéneos de Poisson. NDVI: índice normalizado de vegetación, DISTAD: distancia al adulto más cercano, NORTH: $\cos$ (exposición* $\pi / 180$ ), EAST: sen (exposición * $\pi / 180$ ), DEM: elevación, TWI: índice de humedad topográfica, DENAD: densidad de individuos adultos. Se muestran el índice de Akaike (AIC), el coeficiente de correlación entre lo observado y lo predicho (r). $(*)$ corresponde al modelo seleccionado.

TABLE I. Evaluation of in-homogeneous Poisson models. NDVI: Normalized difference vegetation index, DISTAD: distance to nearest adult, NORTH: $\cos ($ aspect* $\pi / 180)$, EAST: sen (aspect * $\pi / 180)$, DEM: elevation, TWI: topographic wet index, DENAD: density of adult individuals. We show the Akaike index (AIC), the correlation coefficient between observed and predicted values (r). $\left({ }^{*}\right)$ Selected model.

\begin{tabular}{lll}
\hline MODELOS & AIC & $(\mathrm{r})$ \\
\hline$\sim$ NDVI & 3493 & 0.640 \\
$\sim$ DISTAD & 3465 & 0.671 \\
$\sim$ NORTH & 3512 & 0.634 \\
$\sim$ DEM & 3512 & 0.630 \\
$\sim$ EAST & 3512 & 0.630 \\
$\sim$ TWI & 3512 & 0.626 \\
$\sim$ DENAD & 3481 & 0.655 \\
$\sim$ NDVI + DISTAD + NORTH + DEM + EAST + TWI + DENAD & 3432 & 0.698 \\
$\sim$ NDVI + DISTAD + NORTH + EAST + TWI + DENAD & 3432 & 0.706 \\
$\sim$ NDVI + DISTAD + EAST + TWI + DENAD & 3440 & 0.626 \\
$\sim$ NDVI + DISTAD + EAST + DENAD & 3438 & 0.627 \\
$\sim$ DISTAD + EAST + DENAD & 3462 & 0.623 \\
$\sim$ NDVI + EAST + DENAD & 3444 & 0.617 \\
$\sim$ NDVI + DISTAD + DENAD & 3437 & 0.624 \\
$\sim$ NDVI + DISTAD + EAST & 3441 & 0.629 \\
$\sim$ NDVI + DISTAD + NORTH (*) & $\mathbf{3 4 2 8}$ & $\mathbf{0 . 7 1 0}$ \\
$\sim$ EAST + DENAD & 3474 & 0.617 \\
$\sim$ EAST + DISTAD + NDVI & 3441 & 0.629 \\
\hline
\end{tabular}

\section{DISCUSIÓN}

INTERACCIÓN ESPACIAL DE INDIVIDUOS EN DIFERENTES ESTADOS DE DESARROLLO

En la regeneración de $J$. chilensis se observa un patrón de distribución espacial que difiere significativamente de la aleatoriedad. Se constata un patrón agrupado para todos los rangos de distancia analizado (Maltez-Mouro et al. 2007). Los patrones espaciales agrupados de regeneración pueden deberse a procesos endógenos (e.g. dispersión de semillas) o a procesos exógenos, en donde la regeneración responde a otros factores que se producen de manera agrupada (e.g. depresiones en el terreno, parches de bosque, sitios con mayor concentración de humedad) (Austin 2002). Las parcelas analizadas son altamente heterogéneas, específicamente en cuanto a la cobertura vegetacional, característica distintiva de las exposiciones norte de los bosques esclerófilos, donde se puede observar un mosaico de zonas con alta cobertura vegetacional y suelos desnudos (Fuentes et al. 1984). Por otra parte, debido al mecanismo de dispersión de semillas por gravedad, se puede inferir grandes concentraciones de semillas a cortas distancias de la planta madre. Ambos factores pueden estar dando cuenta del patrón agrupado de distribución, o bien, por la alta dependencia de las plántulas por un sotobosque que las proteja (Cabello 2006, Marcelo et al. 2006, González et al. 2009). Esto sugiere la generación de núcleos de regeneración debido a la conjugación de condiciones ambientales propicias para el establecimiento 
de nuevos individuos, observado también en otras especies de este bosque (Fuentes-Castillo et al. 2012). Este patrón puede a su vez sugerir la concentración de la regeneración en zonas donde las plántulas logren escapar a la depredación de semillas, desecación y a la herbivoría. Siendo este último uno de los principales factores de mortalidad en las primeras etapas de desarrollo (Marcelo et al. 2006). La distribución agregada de los individuos de regeneración sugiere que no habrían desventajas asociadas a competencia intraespecífica en $J$. chilensis o que esta sería relativamente débil en las primeras etapas de desarrollo. No obstante, como es esperable en estadios de desarrollo más avanzados, se evidencia una distribución más regular de individuos a corta distancia.

El agrupamiento de individuos de regeneración se produciría principalmente a cortas distancias de la planta madre. Este agrupamiento se puede ver acentuado en especies con frutos de gran tamaño, donde la dispersión de sus semillas es esperable sea a cortas distancias (Cousens et al. 2008). En otras especies de palma, con el mismo mecanismo de dispersión en ecosistemas semiaridos, se ha encontrado una mayor densidad de plántulas cercanas a la planta madre, disminuyendo con la distancia (Barot et al. 1999, Wehncke et al. 2009). En J. chilensis, si bien el máximo de densidad de plántulas ocurre cercano a la planta madre, este patrón difiere en cuanto a la densidad de individuos de regeneración predecible por el mecanismo de dispersión de semillas, en donde la mayor concentración de semillas ocurre con una mayor densidad bajo la copa de planta madre, disminuyendo con la distancia. En este caso, se observa que la mayor densidad ocurre en un rango de distancia entre los 5 y $7 \mathrm{~m}$ desde el adulto más cercano. El patrón observado para $J$. chilensis sigue más bien el mecanismo planteado por Janzen (1970), donde el mayor reclutamiento ocurre a distancias intermedias desde la planta madre. Se produciría un punto de equilibrio (máxima densidad de regeneración) entre la curva de dispersión de semillas (decreciente desde la planta madre) y la probabilidad de supervivencia (creciente desde la planta madre).

Se observa un patrón discordante entre la lluvia de semillas esperada y el reclutamiento, proceso similar al observado en Araucaria araucana (Molina) K. Koch (semilla de gran tamaño y dispersión por gravedad), el cual es modelado principalmente por las altas tasas de depredación de semillas a corta distancia de la planta madre (Sanguinetti \& Kitzberger 2009). Al igual que en A. araucana, el patrón espacial de reclutamiento en $J$. chilensis podría ser reflejo de la depredación de semillas por parte de Octodon degus que compone su dieta principalmente de semillas de J. chilensis (Yates et al. 1994). Sin embargo, la extracción comercial de semillas de $J$. chilensis hace suponer un mecanismo conjunto de depredación y extracción cercano a los árboles semilleros, por lo que el reclutamiento estaría respondiendo a este proceso.
J. chilensis tiene una alta capacidad germinativa cercana al $70 \%$. Sin embargo, ésta tendría una latencia profunda ya que este porcentaje es alcanzado a los 21 meses (Cabello 2006), dejando expuestas las semillas a la depredación o colecta por un largo periodo de tiempo en el campo. Debido a esto, es posible que un número reducido de semillas logren escapar a este efecto conjunto de depredación bajo la copa. La sobreexplotación y depredación de semillas, además de moldear la estructura poblacional (Gonzalez et al. 2009) estaría moldeando a su vez la estructura espacial de la población. Por lo tanto, la regeneración de la especie ocurre de manera agrupada posiblemente por un efecto paralelo entre el mecanismo de dispersión de semillas, que naturalmente produciría patrones agrupados, la depredación de las mismas y acentuado por el efecto nodriza producido por la cobertura de árboles y arbustos que incrementa la probabilidad de sobrevivencia de las plantas en su etapa temprana de desarrollo.

VARIACIÓN DE LA DENSIDAD POBLACIONAL EN FUNCIÓN A LA HETEROGENEIDAD AMBIENTAL

La mayor densidad de individuos de regeneración se encontró cercana a los individuos adultos, especialmente bajo la cobertura de la vegetación acompañante. Este resultado es concordante con los patrones de distribución espacial agregada detectados en los análisis anteriores. El efecto nodriza ha sido ampliamente reportado en comunidades de plantas mediterráneas (Gómez-Aparicio et al. 2004), al igual que en los bosque esclerófilos de la zona central de Chile (Fuentes et al. 1984, Holmgren et al. 2000, Newton \& Tejedor 2011, Fuentes-Castillo et al. 2012). La disminución de la temperatura y el aumento de la humedad podrían ser esenciales en la supervivencia de las plántulas en ecosistemas mediterráneos debido a la prolongada sequía de verano (Gómez-Aparicio et al. 2004). En J. chilensis se ha encontrado un efecto positivo de árboles y arbustos acompañantes independiente de la especie. Sólo se encontró que las plántulas bajo Acacia caven y Retanilla trinervis fueron depredadas antes que las ubicadas bajo otras especies, lo que podría ser explicado por un mecanismo de protección de micromamíferos bajo el dosel espinoso, aumentando así la herbivoría (Marcelo et al. 2006). Este efecto facilitador de la cobertura en la supervivencia de J. chilensis se debería principalmente a una menor pérdida de agua por transpiración debido a la menor temperatura en la sombra bajo el dosel (Marcelo et al. 2006). Por otro lado, la relación positiva y significativa entre la densidad de reclutamiento y la exposición topográfica se explica posiblemente debido a la mayor producción de semillas asociada a individuos mayormente expuestos a la radiación solar, la cual podría incluso inhibirse en lugares muy sombríos y húmedos, al igual que en rodales muy densos, ocasionando una mayor disponibilidad de semillas para el reclutamiento en estas condiciones ambientales (Cabello 2006). 
Los resultados muestran que el modelo de regeneración seleccionado predice correctamente la presencia/ausencia de individuos en aproximadamente el $70 \%$ de los casos. Sin embargo, el porcentaje de variación de la densidad explicada por el modelo fue sólo de un $20 \%$. Esta discordancia sugiere que a pesar de un valor predictivo alto, hay otros factores ecológicos, no considerados en este estudio, que también pueden explicar la densidad poblacional de regenerantes: la masiva colecta de semillas y la depredación de semillas/ plántulas (Marcelo et al. 2006). Este último efecto es muy relevante pues genera desviaciones importantes de la estructura de tamaños de las poblaciones (escasez de plantas jóvenes). Sin embargo, no existen evaluaciones de sus efectos, lo cual no permite tener una comprensión más profunda del futuro de estas poblaciones. Estos aspectos pueden resultar difícil de predecir teniendo en cuenta la poca regulación sobre la extracción de semillas y el efecto de Oryctolagus cuniculus (Simonetti 1989), uno de los principales responsables de la herbivoría de plántulas en los bosques mediterráneos de Chile central (Fuentes et al. 1984, Newton \& Tejedor 2011) y también para esta especie (Fleury et al. 2015).

El establecimiento se maximiza a una distancia intermedia de los individuos adultos (con una mayor probabilidad bajo arbustos nativos) en la cual se pueden estar generando zonas protegidas de los depredadores cerca de las plantas madres. La estructura espacial de la regeneración podría estar modelada por la fuerte presión extractiva que existe sobre las semillas. Este trabajo confirma la facilitación de $J$. chilensis por la vegetación acompañante en su ciclo reproductivo. Por lo cual, la restauración de la especie debe necesariamente considerar la restauración del bosque esclerófilo y la regulación de las actividades extractivas de semillas de la especie.

\section{AGRADECIMIENTOS}

Alejandro Miranda Cerpa agradece a CONICYT / Programa de Doctorado Nacional/21140409, Chile. Agradece a su vez a Antonio Rivera, Raúl Orrego, Cecilia Smith-Ramírez por sus aportes en diferentes etapas de la elaboración de este trabajo. A Paulina González por las fotografías. Al Instituto de Ecología y Biodiversidad (IEB), proyecto ICM P005 - 02.

\section{BIBLIOGRAFÍA}

Alameda, D., R. Villar \& J. M. Iriondo. 2012. Spatial pattern of soil compaction: Trees' footprint on soil physical properties. Forest Ecology and Management 283:128-137.

Austin, M.P. 2002. Spatial prediction of species distribution: an interface between ecological theory and statistical modelling. Ecological Modeling 157: 101-118.

Baddeley, A. \& R. Turner. 2005. Spatstat: an R package for analyzing spatial point patterns. Journal of Statistical
Software 12(6):1-42.

Barot, S., J. Gignoux \& J.C. Menaut. 1999. Demography of a savanna palm tree: predictions from comprehensive spatial pattern analysis. Ecology 80: 1987-2005.

Besag, J. 1977. Contribution to the discussion of Dr. Ripley's paper. Journal of the Royal Statistical Society 39 (2): 193195.

Beven, K.J. \& M.J. KirkBy. 1979. A physically based, variable contributing area model of basin hydrology. Hydrological Sciences Journal 24: 43-69.

Cabello, A. 2006. Jubaea chilensis (Mol.) Baillon. En: C. Donoso (ed.), Las Especies arbóreas de los Bosques Templados de Chile y Argentina. Autoecología. Marisa Cúneo Ediciones, Valdivia, Chile. 678 pp.

Cousens, R., C. Dytham \& R. Law. 2008. Dispersal in Plants. A population perspective. Oxford University Press, New York, USA. 221pp.

Donoso, P. \& R. Nyland. 2005. Seedling density according to structure, dominance and understory cover in old-growth forest stands of the evergreen forest type in the coastal range of Chile. Revista Chilena de Historia Natural 78(1): 51-63.

ERDAS, Inc. 2002. IMAGINE OrthoBase Pro User's Guide ERDAS IMAGINE Version 8.6 (Erdas, Inc. Atlanta, GA).

Ferreira, J.N., M. Bustamante, D.C. García-Montiel, K. Caylor \& E.A. DAvidson. 2007. Spatial variation in vegetation structure coupled to plant available water determined by two-dimensional soil resistivity profiling in a Brazilian savanna. Oecologia 153(2): 417-430.

Fleury, M., W. Marcelo, R.A. VÁsquez, L.A. GonzÁlez \& R.O. Bustamante. 2015. Recruitment Dynamics of the Relict Palm, Jubaea chilensis: Intricate and Pervasive Effects of Invasive Herbivores and Nurse Shrubs in Central Chile. PLOS one 10(7): 1-13.

Fuentes, E.R., R.D. Otaiza, M.C. Alliende, A.J. Hoffmann \& A. PoiAni. 1984. Shrub clumps of the Chilean matorral vegetation: structure and possible maintenance mechanisms. Oecologia 62: 405-411.

Fuentes, E.R., A. Hoffmann, A. Poiani \& M. Alliende. 1986. Vegetation change in large clearings: patterns in the Chilean matorral. Oecologia 68: 358-366.

Fuentes-Castillo, T., A. Miranda, A. Rivera-Hutinel, C. SmithRamírez \& M. Holmgren. 2012. Nucleated regeneration of semiarid sclerophyllous forests close to remnant vegetation. Forest Ecology and Management 274: 38-47.

Getzin, S., T. Wiegand \& S.P. HubBell. 2014. Stochastically driven adult-recruit associations of tree species on Barro Colorado Island. Proceeding of the Royal Society B 281:9-22.

Gómez-Aparicio, L. 2008. Spatial patterns of recruitment in Mediterranean plant species: linking the fate of seeds, seedlings and saplings in heterogeneous landscapes at different scales. Journal of Ecology 96: 1128-1140.

Gómez-Aparicio, L., R. Zamora, J.M. Gómez, J.A. Hódar, J. Castro \& E. Baraza. 2004. Applying plant facilitation to forest restoration in Mediterranean ecosystems: a metaanalysis of the use of shrubs as nurse plants. Ecological Application 14: 1128-1138.

GonzÁlez, L.A. \& A. Vita. 1987. Palma: uso, regeneración y tratamientos. Revista del Campo 11(569): 14-15.

González, L.A., R. Bustamante, C. Navarro, M. Rafael, M. 
Herrera \& M.A. Toral Ibáñez. 2009. The Ecology and management of the chilean palm (Jubaea chilensis (Mol.) Baillon): History, current situation and perspectives. Palms 53(2): 68-74.

Hamill, D. \& S.J. Wright. 1986. Testing the dispersion of juveniles relative to adults: a new analytic method. Ecology 67: 952957.

Hechenleitner, P., M. Gardner, P. Thomas, C. Echeverría, B. Escobar, P. Brownless \& C. Martínez. 2005. Plantas amenazadas del centro-sur de Chile. Distribución, conservación y propagación. Trama Impresores. Valdivia $188 \mathrm{pp}$.

Holmgren, M., M. Scheffer \& M.A. Huston. 1997. The interplay of facilitation and competition in plant communities. Ecology 78: 1966-1975.

Holmgren, M., A.M. Segura, E.R. Fuentes. 2000. Limiting mechanisms in the regeneration of the Chilean matorral: experiments on seedling establishment in burned and cleared mesic sites. Plant Ecology 147: 49-57.

JANZEN, D.H. 1970. Herbivores and the Number of Tree Species in Tropical Forests. American Naturalist 104(940): 501-527.

Jordano, P., P. Pulido, J. Arroyo, J.L. García-Castaño \& P. García-Fayos. 2004. Procesos de limitación demográfica. En: F. Valladares (ed.), Ecología del bosque mediterráneo en un mundo cambiante. Ministerio de Medio Ambiente, Madrid, pp 229-248.

Krause, K. 2003. Radiance conversion of Quickbird data. Technical note. DigitalGlobe Inc. Longmont, Colorado. USA. 4 pp.

LaW, R., D.W. Purves, D.J. Murrell \& U. Dieckman. 2001. Causes and effects of small-scale spatial structure in plant populations. In: J. Silvertown \& J. Antonovics (eds.), Integrating Ecology and Evolution in a Spatial Context pp. 21-44. Blackwell Science, Oxford.

Law, R.J., D.F. Illian, R.P. Burslem, G. Gratzer, C.V.S. Gunatilleke \& I.A.U.N. Gunatilleke. 2009. Ecological information from spatial patterns of plants: insights from point process theory. Journal of Ecology 97(4): 616-628.

Lillesand, T., R. Kiefer \& J. Chipman. 2008. Remote sensing and image interpretation. John Wiley \& Sons, New York, USA. Sixth edition. 756 pp.

Maltez-Mouro, S., L.V. García, T. Marañón \& H. Freitas. 2007. Recruitment patterns in a Mediterranean oak forest: a case study showing the importance of the spatial component. Forest Science 53: 645-652.

Marcelo, W., R. Bustamante \& R. Vásquez. 2006. Efectos de la herbivoría, el microhábitat y el tamaño de las semillas en la sobrevivencia y crecimiento de plántulas de la palma chilena. Revista Ambiente y Desarrollo 22(2): 55-62.

MicheA, G. 1988. Estudio poblacional de palma chilena (Jubaea chilensis) en el sector Ocoa, Parque Nacional de la Campana. Medio Ambiente 9(1): 124-130.

Nathan, R. \& H. Muller-Landau. 2000. Spatial patterns of seed dispersal, their determinants and consequences for recruitment. Trends in Ecology \& Evolution 15(7): 278-285.

Newton, A.C. \& N. Tejedor. 2011. Principios y práctica de la restauración del paisaje forestal: Estudios de caso en las zonas secas de América Latina. UICN. Gland, Suiza. 409 pp.

O’Loughlin, E.M. 1981. Saturation regions in catchments and their relations to soil and topographic properties. Journal of Hydrology 53: 229-246.

Palik, B., R.J. Mitchell, S. Pecot \& M. Battaglia. 2003. Spatial distribution of overstory retention influences resources and growth of longleaf pine seedlings. Ecological Application 13: 674-686.

Pelissier, R. \& F. Goreaud. 2001. A practical approach to the study of spatial structure in simple cases of heterogeneous vegetation. Journal of Vegetation Science 12: 99-108.

Penttinen, A. \& D. Stoyan. 2000. Recent Applications of Point Process Methods in Forestry Statistics. Statistical Science 15(1): 61-78.

Quintanilla, V. \& M. Castillo. 2009. Degradación de Ecosistemas de la palma más Austral del Mundo (Jubaea chilensis) acelerada por los Fuegos Estivales en los cordones litorales de Valparaíso y Viña del Mar $\left(32^{\circ} 50^{\prime}-33^{\circ} 02^{\prime}\right.$ S $)$. Un caso sostenido de perturbación del paisaje. Investigaciones Geográficas 41: 41- 60.

RiPLEy, B.D. 1981. Spatial Statistics. John Wiley \& Sons. Hoboken, New Jersey. 252 pp.

Rozas, V. \& J.J. CAmarero. 2005. Técnicas de análisis espacial de patrones de puntos aplicadas en ecología forestal. Investigación Agraria: Sistemas y Recursos Forestales 14(1): 79-97.

SAnguinetti, J. \& T. Kitzberger. 2009. Araucaria araucana temporal and spatial seedling establishment patterns: masting, seed predation and understory vegetation effects. Revista Chilena de Historia Natural 82: 319-335.

Schulz, J., L. Cayuela, C. Echeverría, J. Salas, J.M. Rey Benayas. 2010. Monitoring land cover change of the dryland forest landscape of Central Chile (1975-2008). Applied Geography 30: 436-447.

Simonetti, J.A. 1989. Microhabitat use by Oryctolagus cuniculus in central Chile: a reassessment. Mammalia 3: 363-368.

Wehncke, E., V.X. López-Medellín \& E. Ezcurra. 2009. Blue fan palm distribution and seed removal patterns in three desert oases of northern Baja California, Mexico. Plant Ecology 208: 1-20.

Wiegand, T. \& K. A. Moloney. 2004. Rings, circles, and nullmodels for point pattern analysis in ecology. Oikos 104: 209-229.

Wilson, J.P. \& J.C. Gallant. 2000. Secondary topographic attributes. En: J.P. Wilson \& J.C. Gallant (eds.), Terrain Analysis: Principles and Applications. pp. 87-131. John Wiley and Sons, New York,

Yates L.R., F. Saiz \& S. Zunino. 1994. Octodon degus: valor nutricional y preferencia del recurso trófico en el Palmar de Ocoa, Parque Nacional La Campana, Chile. Revista Chilena de Historia Natural 67: 89-99.

Zhang, L., T. Luo, X. Liu \& G. Kong. 2010. Altitudinal variations in seedling and sapling density and age structure of timberline tree species in the Sergyemla Mountains, southeast Tibet. Acta Ecologica Sinica 30: 76-80. 